\title{
Rapid progress for non-nuclear estrogen receptor signaling
}

\author{
Michael E. Mendelsohn and Richard H. Karas
}

Molecular Cardiology Research Institute, Tufts Medical Center, Boston, Massachusetts, USA.

\begin{abstract}
Estrogen receptors are best known as ligand-activated transcription factors that regulate vascular cell gene expression. For many years now, a rapid signaling pathway mediated by cell membrane-associated estrogen receptors also has been recognized, but the physiological relevance of this pathway has remained unclear. In this issue of the JCI, Chambliss et al. provide new data to indicate that activation of non-nuclear estrogen receptor signaling regulates processes central to cardiovascular health and disease. These investigators show that an estrogen-dendrimer conjugate (EDC), which activates estrogen receptors but remains non-nuclear, stimulates vascular EC migration in vitro and protects against vascular injury in vivo. They show further that the vascular benefits of EDC in vivo occur selectively in the vasculature, without stimulating the uterus or enhancing growth of breast cancer xenografts. Taken together, these findings indicate that activation of non-nuclear estrogen receptor signaling regulates vascular events of physiological relevance and suggest that translation of these findings into clinically relevant therapeutic interventions is a logical next goal.
\end{abstract}

Estrogen regulates multiple processes in cardiovascular cells and tissues, and abundant evidence gathered over the past three decades indicates that endogenous estrogen in women has cardiovascular protective effects (1). Much of this evidence has been obtained from the study of animal models of cardiovascular disease (CVD), in which estrogen has been found to provide protection, and from observational human studies that show an association between hormone therapy (a combination of estrogen and progesterone for the treatment of perimenopausal symptoms) in postmenopausal women and reduced CVD risk (1). Together, these data gave rise to the hypothesis that estrogen therapy will reduce a postmenopausal woman's risk of developing CVD (1). However, recent randomized clinical intervention studies of hormone therapies in postmenopausal women have failed to demonstrate an overall reduction in CVD risk and instead showed increased risk of stroke and increased CVD events for subgroups of women, especially those who were older and those with a long hormone-free interval (2-4).

Conflict of interest: The authors have declared that no conflict of interest exists.

Citation for this article: J Clin Invest. 2010; 120(7):2277-2279. doi:10.1172/JCI43756.
This discordance between the findings of the randomized clinical trials and the observational and animal data has caused considerable controversy and confusion for both the public and practitioners, with much current discussion focusing on whether the timing of initiation of hormone therapy in relation to the onset of menopause alters its cardiovascular effects (5). The cardiovascular effects of hormone therapy are clearly complex and include a mixture of both potentially beneficial and detrimental effects, underscoring the need for greater clarity about the molecular mechanisms through which estrogen regulates specific cardiovascular processes. The ultimate goal is to develop novel hormone therapies for women that capture the beneficial cardiovascular effects of the hormone while minimizing its potential for harm. To do so, additional studies of estrogen receptor-mediated (ER-mediated) signaling in blood vessels are needed, and the study in this issue of the JCI by Chambliss and colleagues provides just such a study and an important next step (6).

\section{Estrogen receptor actions in the cardiovascular system}

As in most tissues studied, estrogen activates multiple molecular signaling pathways in the cells and tissues of the cardio- vascular system (Figure 1). Functional ERs are expressed in vascular endothelial and smooth muscle cells, and in the cardiomyocytes of the heart (7). ERs, like all steroid hormone receptors, function classically in the nucleus as ligand-activated transcription factors that regulate gene expression in response to hormone binding. ERs can also regulate vascular cell gene expression in the absence of estrogen following activation by growth factor signaling pathways, a process referred to as ligandindependent transcriptional activation. In addition, a third, intriguing mechanism of action of ligand-activated ERs has been studied for many years: the ability of ERs to signal rapidly, in an apparently non-nuclear manner, resulting in downstream activation of specific kinases and their effector molecules (Figures 1 and 2). Rapid, non-nuclear ER signaling in the cardiovascular system has been best studied in ECs, where estrogen and ERs lead to the rapid activation of eNOS, production of nitric oxide, and vasodilation (8). Over the past 15 years, a number of laboratories have explored the molecular events in this fascinating pathway in vascular ECs, in which a subpopulation of ERs localized to cell membrane-based caveolae (small invaginations of the cell membrane) mediate rapid (15-30 minutes), estrogeninduced activation of eNOS via a pathway involving activation of the low-molecularweight $G$ protein $G_{\alpha i}$, the tyrosine kinase src, and the serine/threonine kinases Akt and MAPK (Figure 2). ERs localized to EC caveolae directly bind to a scaffold protein called striatin, which also binds to caveolin-1, the major structural protein of caveolae; striatin is required for estrogenER activation of the rapid, non-nuclear signaling pathway (9).

\section{New evidence for the in vivo relevance of rapid, non-nuclear ER signaling}

In this issue of the JCI, Chambliss and coworkers demonstrate that activation of rapid, non-nuclear ER signaling is 


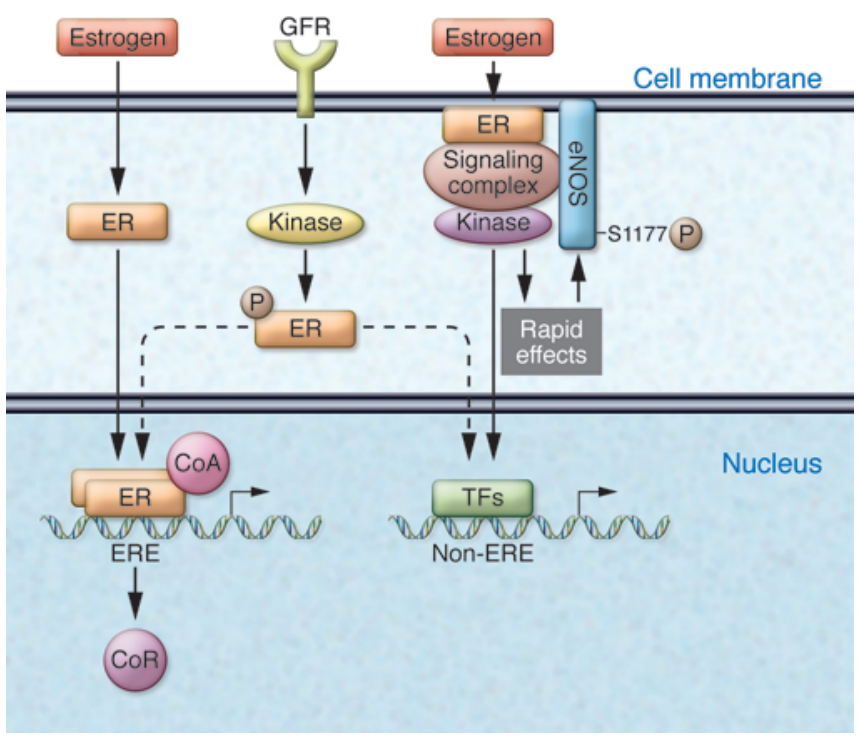

\section{Figure 1}

ER signaling pathways in vascular ECs. ERs are ligand-activated transcription factors that translocate to the nucleus upon estrogen binding and regulate gene expression. Classically, the hormone-bound receptor dimerizes and binds to specific DNA sequences called estrogen response elements (ERE), displacing corepressors (CoR) from the DNA, recruiting coactivator ( $\mathrm{CoA})$ proteins, and activating gene expression. ERs can also be transcriptionally activated via ligand-independent pathways in which growth factor receptor (GFR) activation leads to activation of specific kinases that directly phosphorylate the ER, again leading to altered gene expression, either directly by the ER or via ER interactions with other transcription factors (TFs). In the current issue of the $\mathrm{JCl}$, Chambliss and colleagues demonstrate the in vivo importance of a long-recognized third signaling pathway mediated by non-nuclear ERs in ECs (6). In this pathway, estrogen induces a subpopulation of cell membrane-associated ERs to form a signaling complex that results in rapid activation of specific kinases, which in turn phosphorylate and enzymatically activate eNOS. important in vivo, providing protection against vascular injury in the mouse (6). These data therefore provide critical new evidence that this non-nuclear ER signaling pathway regulates physiologically relevant processes. Chambliss et al. found that a previously characterized estrogen-dendrimer conjugate (EDC) that activates membrane-associated ERs but is excluded from the nucleus (10), stimulated EC migration and proliferation in vitro similarly to $17 \beta$-estradiol (6). Pharmacologic inhibitors and disrupting peptides were then used in studies that indicate that these rapid effects of estrogen in ECs required direct binding between $E R$ and $G_{\alpha i}$, as well as activation of the tyrosine kinase src and eNOS. In vivo studies were used next to show that EDC was equivalent to $17 \beta$-estradiol in accelerating re-endothelialization of the mouse carotid artery following electrical thermal injury, an effect that was blocked by pharmacologic inhibition of either ER or $G_{\alpha i}$. Finally, vascular protective effects of EDC were elegantly confirmed in vivo in a well-characterized atherosclerosis model of mechanical carotid injury in Apoe-knockout mice. In these important experiments, EDC-mediated protection against vascular injury was observed, although it provided somewhat less protection than did $17 \beta$-estradiol itself.

This new study by Chambliss and colleagues (6) makes several important advances in our understanding of both the cardiovascular effects of estrogen and the rapid estrogen/ER/eNOS signaling pathway. First, and most importantly, the study establishes that activation of nonnuclear ER signaling can have relevant physiologic consequences, with potential clinical implications for the design of a next generation of hormone therapies. Second, the study indicates the in vivo importance of specific molecular components of the rapid, non-nuclear signaling pathway, including $\mathrm{G}_{\alpha \mathrm{i}}$. Third, the study indicates the potential for developing pathway-specific selective ER modulators (SERMs), compounds that can activate

non-nuclear effects of estrogen selectively and in a manner distinct from the classical genomic signaling events mediated by ERs and ER target genes.

In the study by Chambliss et al. (6), EDC was shown clearly to act on non-vascular tissues in a manner distinct from estrogen. EDC lacked direct effects on the human breast cancer cell line MCF-7 and on uterine cell function, in contrast to the pro-proliferative and -migratory effects of EDC observed on ECs. Thus,

\section{Extracellular space}

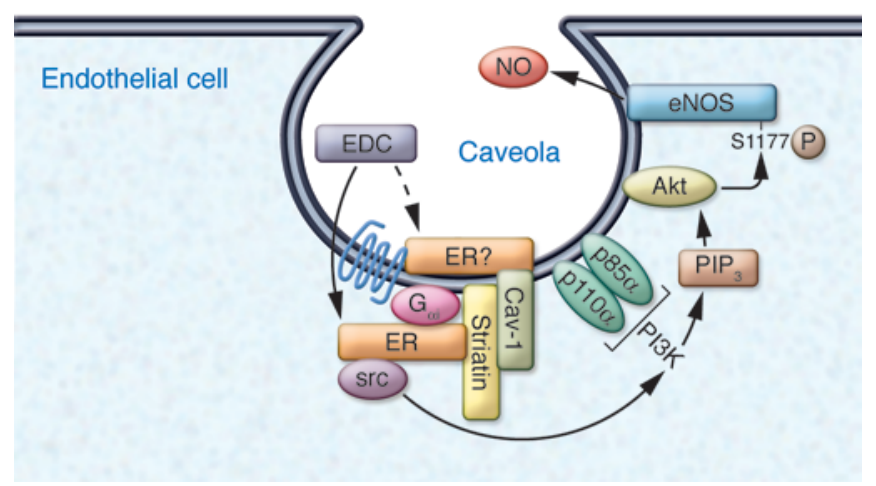

\section{Figure 2}

Non-nuclear ER signaling in vascular EC caveolae. A subpopulation of non-nuclear ERs localize to EC plasma membrane invaginations called caveolae by direct binding to caveolar proteins, including the scaffold protein striatin, which is bound to the major caveolar structural protein, caveolin-1 (Cav-1). Upon estrogen binding, signaling complexes assemble that include ERs and the $G$ protein $G_{\alpha i}$ and sequentially activate the tyrosine kinase src, the serine/threonine kinase $\mathrm{PI} 3 \mathrm{~K}$ that produces phosphatidylinositol $(3,4,5)$-triphosphate $\left(\mathrm{PIP}_{3}\right)$, and the kinase Akt. Akt then directly phosphorylates eNOS on serine 1,177 , leading to its enzymatic activation and production of NO. Whether the ERs are apposed to the inner leaflet of the plasma membrane, or are somehow localized on the external face of the membrane, remains unclear. In the current issue of the $\mathrm{JCl}$, Chambliss and coworkers demonstrate that in vivo activation of this non-nuclear ERdependent pathway by EDC confers protection against vascular injury in the mouse (6). 
non-nuclear ER-mediated signaling initiated by EDC is a cell-specific phenomenon. These findings were confirmed in in vivo experiments, where EDC provided vascular protection but did not stimulate uterotrophic effects or accelerate the growth of breast cancer xenografts as estrogen does. Since unopposed estrogen in humans (i.e., estrogen therapy without concomitant progesterone) is well known to increase the risk of uterine cancer in women, the findings of Chambliss et al. underscore the exciting possibility of developing clinically useful SERMs with tissue-specific actions similar to EDC. The application of the estrogen molecule EDC recently by Katzenellenbogen and colleagues (10) and the recognition of its pathway-specific effects is noteworthy. EDC now emerges as an important step forward in developing tools that allow the non-nuclear effects of ER to be elucidated in a physiological setting and in differentiating these effects from nuclear ER signaling. Identification of therapeutic molecules with vascular protective effects but without effects that stimulate the proliferation of nonvascular cells or the growth of cancer would represent a major advance in translational research for hormone therapies.

\section{Next steps in exploring non-nuclear ER activation}

The current findings thus provide new and important information about nonnuclear ER signaling. They also suggest a number of important questions to be pursued next. The molecular mechanisms that mediate the cell-specific effects of EDC noted above are presently unclear. In MCF-7 cells, EDC activated gene expression, presumably due to activation of transcription factors downstream of the EDC-activated kinases, yet activation of this gene expression program did not enhance MCF-7 cell proliferation or migration (6). Presumably, although this has not yet been shown, transcription factors were also activated in EC downstream of the EDC-activated kinases, yet in these cells, EDC did enhance proliferation and migration (6). What accounts for these cell-specific differences? The molecular mechanisms that mediate these differen- tial responses remain unclear and will be important to understand. Also unclear is the precise location and molecular associations of ER in caveolae and how it is that EDC binds to and activates ER in caveolae. Is the ER apposed to the intracellular side of the caveolar plasma membrane, or is it actually somehow localized on the extracellular side of the plasma membrane (Figure 2)? Many previous investigators have assumed that membrane-associated ERs that mediate rapid effects are intracellular and that estrogen, a highly hydrophobic molecule, diffuses through the plasma membrane in order to gain access to intracellular, membrane-localized ER. Previous confocal microscopic studies of EDC demonstrate both membrane and cytoplasmic (but non-nuclear) localization of a fluorescently labeled version of the compound, but only in ERpositive cells. This distribution has been interpreted to indicate that EDC itself cannot cross the membrane but instead gains access to the cytoplasm only after binding to ER, a model that requires ER to be localized on the extracellular side of the membrane. Such a model is consistent with earlier publications demonstrating recognition of a subset of ER molecules associated with the external cell membrane by a variety of ER-specific antibodies in intact, non-permeabilized cells. However, ER itself has no transmembrane domain, and a plausible mechanism for how ER might be transported to the extracellular membrane surface has not been put forward, nor is one easy to conceptualize.

\section{Concluding remarks}

Although the clinical relevance of these findings remains to be explored, the study by Chambliss et al. (6), clearly establishes proof of concept for the ability of nonnuclear ER signaling by EDC to exert important in vivo physiologic effects. That the majority of efficacious interventions in mouse and animal models have failed to translate into clinically meaningful interventions in humans, underscores the long road ahead required to mature the current findings into novel therapeutic interventions. What this interesting non-nuclear pathway means for human physiology and whether this pathway is relevant to our understanding of the changes occurring with the menopausal transition both remain to be understood. However, the elegant new set of findings provides a critical step forward in translating the many years of study of nonnuclear, rapid hormonal signaling (11) into the possibility of new therapeutic tools for human pathophysiology.

\section{Acknowledgments}

This work is supported in part by NIH grants P01 HL077378 and R01 HL056069 (to M.E. Mendelsohn) and NIH grants R01 HL61298 and R01 HL07803 (to R.H. Karas).

Address correspondence to: Michael E. Mendelsohn or Richard H. Karas, 800 Washington Street, Box \#80, Boston, Massachusetts 02111, USA. Phone: 617.636.8776; Fax: 617.636.1444; E-mail: MMendelsohn@ tuftsmedicalcenter.org (M.E. Mendelsohn); RKaras@tuftsmedicalcenter.org (R.H. Karas).

1. Mendelsohn ME, Karas RH. Mechanisms of disease: the protective effects of estrogen on the cardiovascular system. N EnglJ Med. 1999;340(23):1801-1811.

2. Hsia J, et al. Conjugated equine estrogens and coronary heart disease. Arch Intern Med. 2006;166(3):356-365.

3. Hulley S, et al. Randomized trial of estrogen plus progestin for secondary prevention of coronary heart disease in postmenopausal women. JAMA. 1998;280(7):605-613.

4. Manson JE, et al. Estrogen plus progestin and the risk of coronary heart disease. $N$ Engl J Med. 2003;349(6):523-534.

5. Grodstein F, Clarkson TB, Manson JE. Understanding the divergent data on postmenopausal hormone therapy. N Engl J Med. 2003;348(7):645-650.

6. Chambliss KL, et al. Non-nuclear estrogen receptor $\alpha$ signaling promotes cardiovascular protection but not uterine or breast cancer growth in mice. J Clin Invest. 2010;120(7):2319-2330.

7. Mendelsohn ME, Karas RH. Molecular and cellular basis of cardiovascular gender differences. Science. 2005;308(5728):1583-1587.

8. Mendelsohn ME. Nongenomic, ER-mediated activation of endothelial nitric oxide synthase: how does it work? what does it mean? Circ Res. 2000;87(11):956-960.

9. Qing L, Pallas DC, Surks HK, Baur WE, Mendelsohn ME, Karas RH. Striatin assembles a membrane signaling complex necessary for rapid, nongenomic activation of endothelial NO synthase by estrogen receptor $\alpha$. Proc Natl Acad Sci U S A. 2004;101(49):17126-17131.

10. Harrington WR, et al. Estrogen dendrimer conjugates that preferentially activate extranuclear, nongenomic versus genomic pathways of estrogen action. Mol Endocrinol. 2006;20(3):491-502.

11. Touchette N. Man bites dogma: a new role for steroid hormones. J NIH Res. 1990;2:71-74. 